\title{
Spectrum of Adrenal Lesions on Fine-Needle Aspiration Cytology: A Tertiary Care Centre Experience
}

\author{
Pavneet Kaur $^{1}$ Ankita Soni ${ }^{1}$ Ruchita Tyagi ${ }^{1}$ Harpreet Kaur ${ }^{1}$ Kanwarpal S. Selhi ${ }^{1}$ \\ ${ }^{1}$ Department of Pathology, Dayanand Medical College and Hospital, \\ Ludhiana, Punjab, India \\ Address for correspondence Ankita Soni, MD, Department: \\ Pathology, Institution: Dayanand Medical College and Hospital, \\ Tagore Nagar, Ludhiana, 141001, Punjab, India \\ J Lab Physicians 2022;14:231-236. \\ (e-mail: ankitatavish@gmail.com).
}

\begin{abstract}
Introduction Fine-needle aspiration cytology (FNAC) is an easy, quick, and specialized technique to distinguish neoplastic from non-neoplastic adrenal lesions, yet limited to tertiary care centers. It helps in analyzing symptomatic, as well as incidental adrenal lesions with high sensitivity and specificity.

Aim This study was conducted to determine the cytological spectrum of adrenal lesions in a tertiary care center.

Material and Methods This was a retrospective study which included a total of 19 cases of adrenal FNAC received from June 2017 till June 2019 in a north Indian tertiary care university hospital. All the lesions were broadly classified into non-neoplastic and neoplastic categories. The non-neoplastic lesions were divided into infective causes and cystic lesions. Neoplastic lesions were further grouped into benign and malignant lesions. Immunohistochemical findings were retrieved from the hospital records wherever accessible.

Results A total of 19 cases were aspirated, of which 16 cases (84.20\%) yielded satisfactory material. Six cases $(31.57 \%$ ) showed non-neoplastic pathology of which one was a cystic lesion, three were infective (two histoplasmosis and one tuberculosis), and two showed only benign adrenal cortical cells in a setting of known extra-adrenal primary malignancy. The neoplastic group comprised of 10 cases $(52.63 \%)$ of which 4 cases showed metastatic carcinomatous deposits from a known extra-adrenal primary malignancy and 6 cases showed primary adrenal neoplasm (one case of myelolipoma, one case of pheochromocytoma, and four cases of adrenal neoplasm) which were then subjected to biopsy and immunohistochemistry. A final diagnosis of pheochromocytoma was made in three cases, adrenocortical carcinoma in one case, and one case was

\section{Keywords}

- adrenal neoplasm

- cytology

- immunohistochemistry

inconclusive because of nonrepresentative biopsy.

Conclusion Image-guided fine-needle aspiration cytology of adrenal lesions helps to determine the exact nature of the infection, avoids unnecessary surgery, and helps in targeted management. However, histopathological evaluation with immunohistochemistry remains the diagnostic modality of choice with regard to neoplastic lesions.
\end{abstract}

published online January 18,2022
DOI https://doi.org/

$10.1055 / \mathrm{s}-0041-1741441$ ISSN 0974-2727. (c) 2022. The Indian Association of Laboratory Physicians. All rights reserved.

This is an open access article published by Thieme under the terms of the Creative Commons Attribution-NonDerivative-NonCommercial-License, permitting copying and reproduction so long as the original work is given appropriate credit. Contents may not be used for commercial purposes, or adapted, remixed, transformed or built upon. (https://creativecommons.org/ licenses/by-nc-nd/4.0/)

Thieme Medical and Scientific Publishers Pvt. Ltd., A-12, 2nd Floor, Sector 2, Noida-201301 UP, India 


\section{Introduction}

Image-guided fine-needle aspiration cytology (FNAC) of adrenal lesions is a specialized technique limited to tertiary care centers for analyzing symptomatic, as well as incidental lesions. ${ }^{1}$ FNAC has been proven as a highly sensitive and specific diagnostic tool in most endocrine lesions, particularly thyroid. ${ }^{2}$ Image-guided FNAC in adrenal lesions plays an important role in distinguishing non-neoplastic from neoplastic lesions. It helps in metastatic workup of epithelial malignancies as adrenal is a common site for metastatic carcinomatous deposits. Cytological evaluation of adrenal masses may avoid unnecessary surgical interventions in non-neoplastic conditions. This study evaluated the cytological spectrum of adrenal lesions in a tertiary care health center, as well as the advantages and limitations of adrenal FNAC.

\section{Materials and Methods}

This was a retrospective study conducted in the Department of Pathology from June 2017 till June 2019 at Dayanand Medical College and Hospital (DMC\&H) in Ludhiana, a tertiary care university hospital. All the 19 cases of adrenal FNAC performed in these 2 years were analyzed for the study. Informed prior consent was taken in every case. Ethical clearance was obtained from the institutional ethical committee.

FNAs were performed using a 21- to 23-gauge needle and 20 cc syringe under radiological guidance, followed by rapid onsite evaluation (ROSE) of aspiration samples for adequacy. A total of 6 to 8 smears were made from every case. Air-dried smears were stained with May-Grunwald-Giemsa stain and alcohol-fixed smears were stained with hematoxylin-eosin and Papanicolaou stains. Special stains like 20\% Ziehl-Neelsen (ZN) stain for acid-fast bacilli, periodic acid-Schiff's (PAS), and Gomori's methenamine silver (GMS) stains for fungus were performed when needed. All smears were read and reported by two experienced cytopathologists.

The lesions were broadly classified cytologically into non-neoplastic and neoplastic categories. The non-neoplastic lesions were further divided into infective and benign cystic lesions and the neoplastic lesions were divided into benign and malignant neoplasm. The malignant neoplasm included both primary and metastatic malignancies. No major complication was encountered in any of the cases. Clinical and radiological profile was retrieved from hospital records. Tru-cut biopsy and immunohistochemical findings were also obtained from the hospital record wherever available.

\section{Statistical Analysis}

SPSS statistics 16.0 for windows (Chicago, Illinois, United States) was used for statistical analysis of data. Continuous data were represented as mean \pm standard deviation (SD) or median with interquartile range if it was distributed normally or skewed, respectively. The categorical data were represented as percentages.

\section{Results}

A total of 19 image-guided FNACs were performed during the study period of which 9 were male and 10 were female patients. Age varied between 14 and 75 years with a mean age of 53 years. Pain abdomen was the most common presenting symptom with fever being the next common.

Radiologically, bilateral adrenal involvement was identified in five cases of which two were diagnosed as histoplasma infection, two as metastatic carcinomatous deposits from a known extra-adrenal primary malignancy of pancreatic neuroendocrine carcinoma, and endometrioid adenocarcinoma while satisfactory material could not be yielded in the fifth case. Space occupying lesion/lesions were detected in nine cases. The adrenal lesion size ranges from 17 to $60 \mathrm{~mm}$. In this study, the number of FNAs performed on the right adrenal gland $(n=10)$ was slightly higher than that on left $(n=9)$. The clinical, radiological, and pathological profile of all the study patients has been described in - Table 1 .

Out of 19 cases, 3 (15.78\%) were unsatisfactory because of insufficient cellularity. Of which two were incidentally detected on radiology as diffuse glandular enlargement and one was given a provisional radiological diagnosis of adrenal cortical adenoma on computed tomography (CT). Two to three passes were given for each of these cases. Further passes could not be performed as all three patients refused for the same.

A satisfactory material was obtained in 16 cases (84.20\%), of which 6 (31.57\%) turned out to be non-neoplastic and the remaining 10 (52.63\%) were neoplastic.

Of the non-neoplastic lesions, one was a benign cystic lesion, three were infective (two histoplasmosis and one tuberculosis), and two cases where FNAC of the adrenal gland was done as a part of metastatic workup showed only benign adrenal cortical cells with no malignant cells in a setting of a known extra-adrenal primary malignancy of breast (invasive ductal carcinoma) and stomach (adenocarcinoma) which were diagnosed on histopathology of the primary malignant lesion. One of the two histoplasmosis cases was a known diabetic with liver cirrhosis while the other had no comorbidity. On cytomorphology, both the cases showed the presence of extracellular, as well as intracellular (within the macrophages) organisms which were ovoid to spherical measuring 2 to $4 \mu \mathrm{m}$ and surrounded by a clear halo ( - Fig. 1A,B). The only case of adrenal tuberculosis found in this study was a known case of pulmonary tuberculosis and was on antitubercular therapy (ATT). Cytomorphology showed the presence of epithelioid cell granulomas with giant cells and 20\% ZN stain was noncontributory as the patient was on ATT.

Out of the neoplastic lesions, one was reported as myelolipoma, cytological examination of which showed the presence of mature adipose tissue fragments admixed with hematological elements such as megakaryocytes, nucleated red blood cells, and lymphocytes (-Fig. 1C,D). However, a biopsy was not performed for this case.

A cytological diagnosis of pheochromocytoma was given in one case, the FNAC of which showed tissue fragments with 

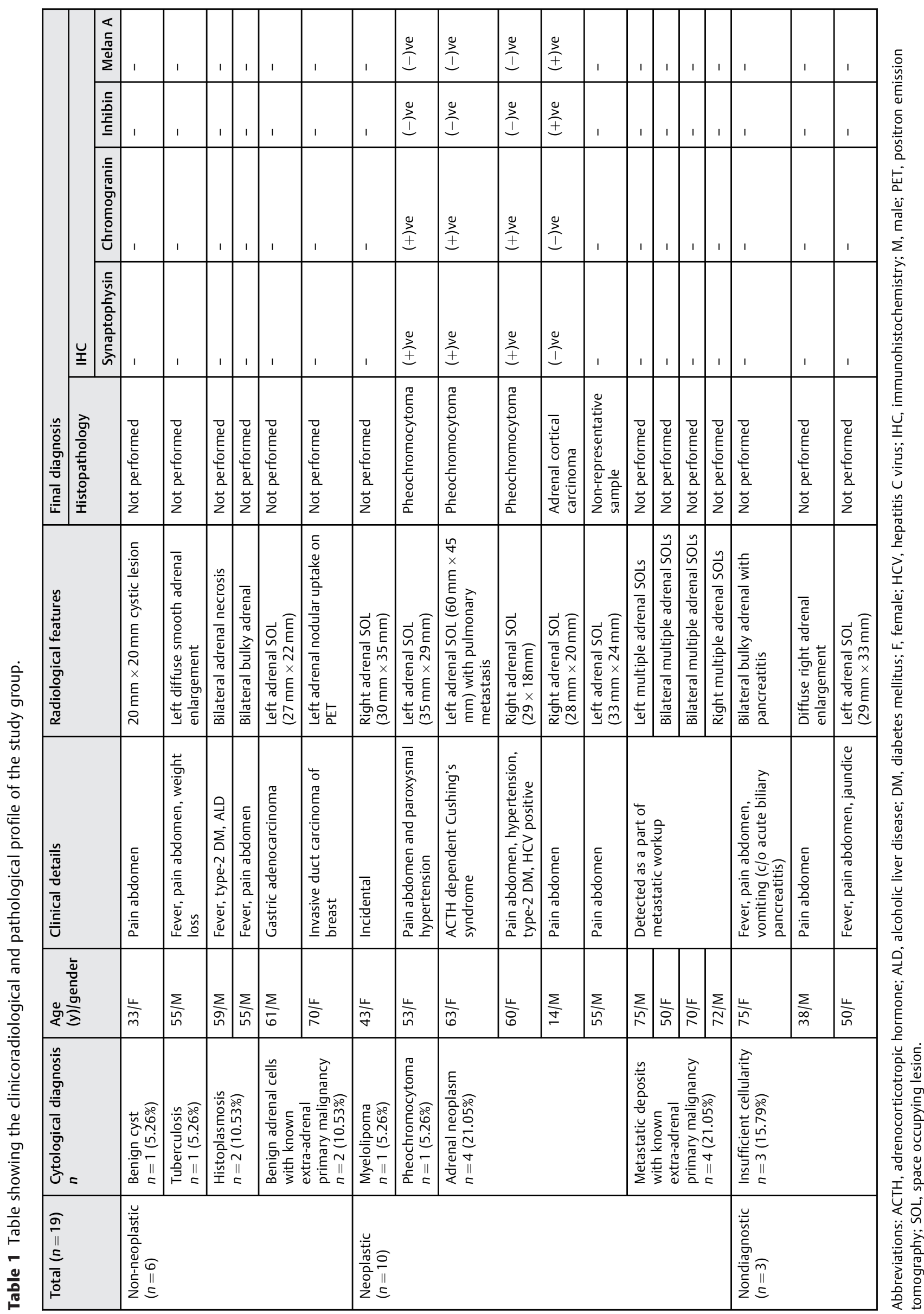


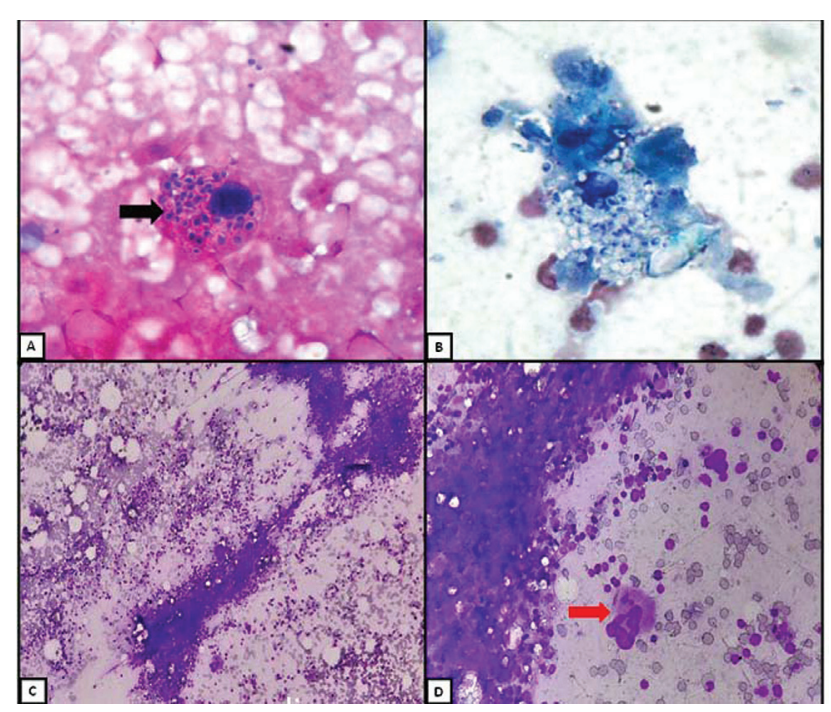

Fig. 1 (A) A macrophage showing intracellular histoplasma organism (black arrow) on H\&E stain $(\times 400)$ and $(B)$ Papanicolaou stain $(\times 400)$. FNA features of myelolipoma showing adipose tissue fragments and bone marrow elements seen as megakaryocyte (red arrow) and nucleated red blood cells on Giemsa's stain at (C) $\times 100$ and (D) at $\times 400$. FNA, fine-needle aspiration; $H \& E$, hematoxylin and eosin.

endothelial cell rimming. The tumor cells displayed salt and pepper chromatin and a moderate amount of cytoplasm. Histopathological examination showed tumor cells having salt and pepper chromatin, arranged in zellballen pattern. On immunohistochemistry (IHC), the tumor cells expressed positivity for chromogranin A and synaptophysin.

In four cases of adrenal neoplasm, definite type of the neoplasm could not be ascertained and a cytological diagnosis of primary adrenal neoplasm could only be rendered. The final diagnosis was made on histopathology and IHC in these cases.

A cytological diagnosis of metastatic deposits from a known extra-adrenal primary malignancy which had been diagnosed on histopathology and IHC of the primary lesion (colon adenocarcinoma, pancreatic adenocarcinoma, nonHodgkin's lymphoma of cecum, and endometrioid adenocarcinoma) was given in four cases. Adrenal FNAC was performed as a part of metastatic workup and no biopsy/IHC was available in any of these cases. On cytology, the adenocarcinomatous deposits appeared as tumor cells arranged in glandular configuration, while the lymphomatous deposit was seen as singly scattered medium to large cells exhibiting an altered N/C ratio, prominent nucleoli in few, and scant amount of cytoplasm with brisk mitosis and lymphoglandular bodies. All these cases showed multiple lesions in adrenal (either unilateral or bilateral) which in conjunction with cytomorphology favored a diagnosis of metastasis over a primary adrenal neoplasm.

True-cut biopsy and IHC were available in the cases with cytological diagnosis of pheochromocytoma $(n=1)$ and primary adrenal neoplasm $(n=4)$ which helped in further subtyping of adrenal neoplasm in these cases. The cytological diagnosis of pheochromocytoma was confirmed on histology and a final diagnosis of pheochromocytoma was made in three cases of primary adrenal neoplasm, adrenocortical carcinoma in one, and one case was inconclusive because of a nonrepresentative sample.

\section{Discussion}

Image-guided FNAC has been reported as a highly sensitive and specific diagnostic tool in many endocrine organs, especially the thyroid gland. The role of FNAC is increasing in differentiating various adrenal lesions and for staging malignancies, yet limited to tertiary care centres. ${ }^{3}$ Many investigators in the past have reported an inadequacy rate of as high as $37 \%$ with percutaneous image-guided FNAC. ${ }^{4}$ However, in this study, $15.78 \%$ of total cases were found unsatisfactory because of poor cellularity. A combination of experienced radiologists and cytopathologists is the key to better sample yield. In this study, ROSE was done in every case and multiple passes (2-4) were given to minimize the unsatisfactory samples.

Image-guided FNAC can be done either percutaneously (ultrasound/CT guided) or endoscopic ultrasound (EUS) guided. In the present study, one FNAC was performed under EUS guidance, while the rest were percutaneous ultrasound guided. Percutaneous FNAC is an invasive procedure and may impart morbid complications like hemorrhage, pneumothorax, and septicemia in 8 to $13 \%$ of cases. ${ }^{5}$ In this study, no patient experienced any of these complications.

Differential diagnosis of adrenal lesions includes infections, infiltrative disease, adrenal hemorrhage, primary adrenal neoplasm (benign and malignant), and metastases. ${ }^{6} \mathrm{~A}$ combination of clinical data and radiological findings helps to differentiate benign and malignant lesions. However, cytomorphology contributes to determine the exact nature of infection and to differentiate primary adrenal neoplasm from metastases which helps to determine the treatment plan.

Adrenal cystic lesions are rare and mostly discovered incidentally with an incidence rate ranging from 0.064 to $0.18 \%$. However, the detection rate is increasing with technological advances in imaging such as $\mathrm{CT}$ and ultrasonography. It comprises a broad spectrum ranging from pseudocysts to cystic primary adrenal neoplasm. Radiology in conjunction with microscopic examination helps in differentiating the benign from malignant cystic lesions which are vital for targeted therapy. ${ }^{7}$ In this study, one case was diagnosed as a benign cystic lesion which was incidentally detected on abdominal CT and showed no solid component. Cytomorphology showed no malignant cells.

In developing countries like India, tuberculosis remains the most common cause for adrenal insufficiency comprising 46 to $56 \%$ of all cases ${ }^{8,9}$ However, in this study, histoplasmosis was found in two cases while tuberculosis in one case. Special stains are used to confirm the infective pathogen like $20 \%$ ZN stain for mycobacterium tuberculosis and PAS and GMS stain for histoplasma. Infective lesions usually have bilateral diffuse adrenal involvement with heterogeneous echotexture due to necrosis and type-B symptoms. In our study, two out of three infective cases had bilateral adrenal 
involvement with one of the histoplasmosis case showing diffuse necrosis. All three cases presented with fever and pain abdomen. The differential diagnosis of histoplasma includes Leishmania donovani, cryptococcus, histoplasma, and blastomyces. Awareness of clinical and cytomorphological features plays an important role in identifying the infective pathogen and helps in targeted management without the need for unnecessary surgery. ${ }^{10}$

Adrenal myelolipoma is a rare benign tumor with female predominance and is one of adrenal incidentaloma. It can be easily detected on radio imaging, yet microscopic examination confirms the diagnosis. The differential diagnosis includes teratoma, lipoma, liposarcoma, and adrenal neoplasm both primary and metastatic. ${ }^{11}$ In this study, a 43 -year-old female was incidentally diagnosed on CT with a right adrenal lesion (measuring $33 \mathrm{~mm} \times 30 \mathrm{~mm}$ in size). On cytology, it is characterized by mature adipose tissue fragments and scattered hematopoietic elements at varying stages of maturation and histopathology confirms the same. ${ }^{12}$

FNAC is a useful tool in malignant adrenal lesions with a sensitivity of 85 to $93 \%{ }^{13}$ The adrenal gland is one of the most common sites for metastasis. In a study, lung carcinoma was found to be the most common indication for imageguided FNA followed by breast carcinoma and melanoma. ${ }^{14}$ However, in our study, gastrointestinal malignancies were responsible for $66.6 \%$ of adrenal lesions with suspected metastasis followed by endometrioid adenocarcinoma and breast carcinoma. Out of the six suspected cases of adrenal metastasis with known extra-adrenal primary malignancy, two had shown only benign cortical cells while the remaining four had shown metastatic carcinomatous deposits from colon adenocarcinoma, pancreatic adenocarcinoma, nonHodgkin's lymphoma of cecum, and endometrioid adenocarcinoma. Radiologically, bilateral multiple adrenal lesions were seen in two, unilateral multiple lesions in two, and unilateral solitary lesion in two cases.

Adrenal cortical carcinoma (ACC) is a rare primary adrenal tumor (annual incidence of 1.5 to 2 per million) with a bimodal age presentation. The functional ACC may present with features of hyperaldosteronism, virilization, feminization, and Cushing's syndrome. ${ }^{15}$ Diagnosis of ACC should only be made in presence of a cortical nodular lesion as the morphology of normal adrenocortical tissue is indistinguishable from cortical neoplastic lesions. Cytologically, it presents as a cellular tumor with cells arranged in clusters along with individually scattered cells against a lipid-rich necrotic background. The tumor cells show centrally placed hyperchromatic nuclei, occasional prominent nucleoli, and abundant amount of vacuolated cytoplasm ( - Fig. 2A,B $){ }^{16}$ In this study, one case which was initially diagnosed as adrenal neoplasm on cytology was confirmed to be ACC on histopathology and IHC showing immunopositivity for inhibin and melan A. This patient presented with adrenocorticotropic hormone-dependent Cushing's syndrome having uncontrolled diabetes mellitus and hypertension. On CT of abdomen and chest, a $60 \mathrm{~mm} \times 45 \mathrm{~mm}$ space-occupying lesion was seen in the left adrenal with suspected pulmonary metastasis. The cytological differentials of ACC include adre-

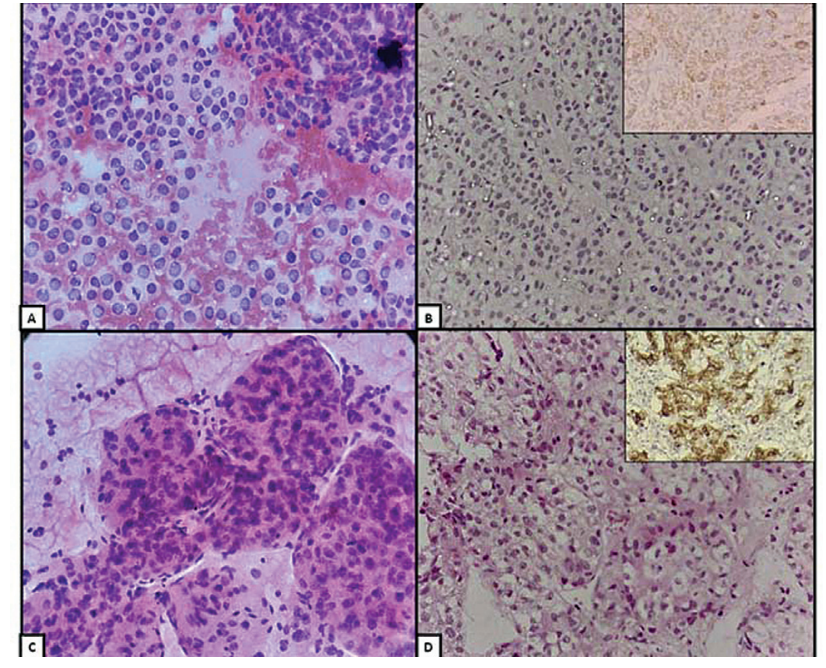

Fig. 2 FNA (A) features of adrenal cortical carcinoma showing tumor cells with centrally placed pleomorphic nuclei, prominent nucleoli, and abundant vacuolated cytoplasm $(\mathrm{H} \& \mathrm{E} ; \times 400)$. Histopathological (B) features of adrenal cortical carcinoma showing tumor cells arranged in trabeculae and sheets $(\mathrm{H} \& \mathrm{E} ; \times 400)$ along with tumor cells showing immunopositivity for inhibin (inset). FNA (C) features of pheochromocytoma showing tissue fragments with endothelial cell rimming. The tumor cells have salt and pepper chromatin and moderate amount of cytoplasm (H\&E; ×400). Histopathological (D) features of pheochromocytoma showing tumor cells arranged in zellballen pattern, having salt and pepper chromatin $(\mathrm{H} \& \mathrm{E} ; \times 400)$ along with tumor cells exhibiting strong cytoplasmic granular positivity for chromogranin A (inset). FNA, fine-needle aspiration, H\&E, hematoxylin and eosin.

nal adenoma, hepatocellular carcinoma, renal cell carcinoma, and metastatic mucinous adenocarcinoma, and metastatic melanoma. There is no definitive feature to diagnose ACC on cytology. Yet, features such as the absence of any other primary site, tumor size $>5 \mathrm{~cm}$, polygonal tumor cells having prominent nucleoli and an abundant amount of granular cytoplasm, a lipid-rich background, brisk mitosis, and necrosis favor ACC. ${ }^{1}$ However, immunopositivity for inhibin, melan A, and calretinin confirms the diagnosis.

Pheochromocytoma is an adrenal medullary neoplasm that commonly occurs in a fourth or fifth decade. It has an incidence rate of 2 to 8 per million. Excess production of catecholamines by the tumor cells may cause hypertensive crisis. ${ }^{15}$ Cytology smears from pheochromocytoma are cellular showing tissue fragments and singly scattered cells with many bare nuclei. Nuclear pleomorphism is readily evident along with salt and pepper chromatin and cytoplasmic metachromatic granules which get highlighted in diff-quik stained smears ( - Fig. 2C,D). ${ }^{17}$ In this study, one case with cytological diagnosis of pheochromocytoma and two cases with a cytological diagnosis of adrenal neoplasm was proven to be pheochromocytoma on histopathology and IHC showing immunopositivity for synaptophysin and chromogranin A. All three cases presented with abdominal pain and space-occupying lesions on CT. Two of these patients presented with uncontrolled hypertension. None of these cases had a syndromic association. The differential diagnosis of pheochromocytoma includes adrenal cortical adenoma, 
ACC, adrenal medullary hyperplasia, metastatic renal cell carcinoma, and metastatic melanoma. Immunopositivity for chromogranin A, synaptophysin, and S-100 confirms the diagnosis of pheochromocytoma.

Though cytology is sensitive to diagnose adrenal neoplasm, yet histopathology with IHC is required for further typing and management of adrenal neoplasms both primary and secondary. The ability to diagnose infective pathology on FNAC decreases unnecessary surgeries and hence reduced morbidity and mortality. This study has a good representation of lesions ranging from cystic lesions to infective pathology to neoplasm, both primary and secondary.

\section{Limitations}

This study has various limitations. First, being a retrospective study carries an inherent bias of study sampling. Second, the sample size of the study is less. Therefore, this limited number of cases could attribute to the disproportionate distribution. Third, a cytohistological correlation could not be done in all the cases as the histopathological examination was not available in the cases of adrenal metastases from a known extra-adrenal primary malignancy.

\section{Conclusion}

This study summarizes a tertiary care center's experience in regard to adrenal FNACs. Image-guided FNAC of adrenal masses in combination with clinical and radiological data are a reliable and safe technique with high diagnostic accuracy. It helps to determine the exact nature of the infection, avoids unnecessary surgery, and helps in targeted management. A combination of experienced radiologists and cytopathologists is essential to get a satisfactory sample. However, cytology alone is insufficient to reach a final diagnosis in neoplastic conditions as it requires histopathological evaluation and IHC.

\section{Funding}

None.

\section{Conflict of Interest}

None declared.

\section{References}

1 Rana C, Krishnani N, Kumari N. Spectrum of adrenal lesions on fine needle aspiration cytology. Indian J Pathol Microbiol 2012;55 (04):461-466

2 Ren R, Guo M, Sneige N, Moran CA, Gong Y. Fine-needle aspiration of adrenal cortical carcinoma: cytologic spectrum and diagnostic challenges. Am J Clin Pathol 2006;126(03):389-398

3 Lumachi F, Borsato S, Brandes AA, et al. Fine-needle aspiration cytology of adrenal masses in noncancer patients: clinicoradiologic and histologic correlations in functioning and nonfunctioning tumors. Cancer 2001;93(05):323-329

4 Tirabassi G, Kola B, Ferretti M, et al. Fine-needle aspiration cytology of adrenal masses: a re-assessment with histological confirmation. J Endocrinol Invest 2012;35(06):590-594

5 Moreira SG Jr., Pow-Sang JM. Evaluation and management of adrenal masses. Cancer Contr 2002;9(04):326-334

6 Mantero F, Arnaldi G. Investigation protocol: adrenal enlargement. Clin Endocrinol (Oxf) 1999;50(02):141-146

7 Kini JR, Gautam K, Augustine A. Adrenal gland cyst: a diagnostic conundrum: report of a case with review of literature. J Med Soc 2014;28:123-124

8 Al-Mamari A, Balkhair A, Gujjar A, et al. A case of disseminated tuberculosis with adrenal insufficiency. Sultan Qaboos Univ Med J 2009;9(03):324-327

9 Kumar R, Dey P. Fine-needle aspiration cytology of non-neoplastic adrenal pathology. Diagn Cytopathol 2016;44(06):472-476

10 Kapatia G, Saha A, Rohilla M, et al. Clinical and morphological spectrum of histoplasmosis on cytology along with the review of literature. Acta Cytol 2020;64(06):532-538

11 Puri S, Mardi K, Gupta N, Rao M. Adrenal incidentalomas with review of literature. J Cytol Histol 2016;7:426

12 Katsuta K, Nakabayashi H, Kuroda Y, Liu PI. Adrenal myelolipoma: preoperative diagnosis by fine-needle aspiration cytology. Diagn Cytopathol 1989;5(03):298-300

13 Katz RL, Shirkhoda A. Diagnostic approach to incidental adrenal nodules in the cancer patient. Results of a clinical, radiologic, and fine-needle aspiration study. Cancer 1985;55(09):1995-2000

14 Welch TJ, Sheedy PF II, Stephens DH, Johnson CM, Swensen SJ. Percutaneous adrenal biopsy: review of a 10-year experience. Radiology 1994;193(02):341-344

15 Karre S, Gorva A, Shanmugam C, Gorrela VDPK, Veeragandham S Histopathologic spectrum of adrenal lesions. Ind J Pathol Oncol 2018;5:463-469

16 Proye CAG, Pattou FN. Adrenocortical carcinoma. Nonfunctioning and functioning In: Clark OH, Duh Q-Y, eds. Textbook of Endocrine Surgery. Philadelphia, PA: WB Saunders; 1997:490-496

17 Jiménez-Heffernan JA, Vicandi B, López-Ferrer P, González-Peramato P, Pérez-Campos A, Viguer JM. Cytologic features of pheochromocytoma and retroperitoneal paraganglioma: a morphologic and immunohistochemical study of 13 cases. Acta Cytol 2006;50(04):372-378 